\title{
Guided modes in non-Hermitian optical waveguides
}

\author{
Elena G. Turitsyna, ${ }^{1,2}$ Ilya V. Shadrivov, ${ }^{2}$ and Yuri S. Kivshar ${ }^{2}$ \\ ${ }^{1}$ Aston Institute of Photonic Technologies, Aston University, Aston Triangle, Birmingham B4 7ET, United Kingdom \\ ${ }^{2}$ Nonlinear Physics Centre, Australian National University, Canberra, Australian Capital Territory 2601, Australia \\ (Received 7 April 2017; published xxxxxx)
}

\begin{abstract}
We study guided modes in non-Hermitian optical waveguides with dielectric layers having either gain or loss. For the case of a three-layer waveguide, we describe stationary regimes for guided modes when gain and loss compensate each other in the entire structure rather than in each layer. We demonstrate that, by adding a lossless dielectric layer to a double-layer waveguide with the property of parity-time $(\mathcal{P} \mathcal{T})$ symmetry, we can control a ratio of gain and loss required to support propagating and nondecaying optical guided modes. This novel feature becomes possible due to the modification of the mode structure, and it can allow using materials with a lower gain to balance losses in various optical waveguiding structures. In addition, we find a non- $\mathcal{P} \mathcal{T}$-symmetric regime when all guided modes of the system have their losses perfectly compensated.
\end{abstract}

DOI: 10.1103/PhysRevA.00.003800

56 the conventional point of view, it is reasonable to expect that

57 by adding gain to the waveguiding structure one can control the characteristics of the propagating modes, as was shown in 58 Ref. [15]. In plasmonic structures, waveguiding is suppressed 59 by losses particularly strongly. There is a search in either 60 optimizing the geometry for these structures [16] or using 61 novel materials [17]. Clearly, such approaches try to minimize 62 losses, and one needs gain materials to compensate losses in 63 plasmonic structures (see, e.g., Refs. [18-21]).

Recently, Suchkov et al. [22] investigated pseudo- 65 Hermitian (PH) optical couplers and compared their properties 66 with those of $\mathcal{P} \mathcal{T}$-symmetric couplers. They revealed that ${ }_{67}$ the mode spectrum can be entirely real even without $\mathcal{P} \mathcal{T}{ }_{68}$ symmetry, provided the waveguides in a coupler are placed 69 in a special order. Being inspired by those findings, here we 70 study three-layer non-Hermitian dielectric waveguides with 71 gain and/or loss (e.g., those shown in Fig. 1). We choose 72 the three-layer structure since the additional parameters allow 73 one to achieve a wider range of regimes as compared to 74 two-layer structures, which were mostly studied up to now. 75 For the case of three-layer waveguides, we describe the 76 stationary regimes when gain and loss compensate each other 77 globally but not locally. We reveal that this system, even being 78 non- $\mathcal{P} \mathcal{T}$ symmetric, supports different types of asymmetric 79 modes and allows additional functionalities and control of the 80 guided modes. We believe that our approach can be useful 81 for reducing the value of gain for balancing losses in optical 82 waveguides.

\section{THREE-LAYER WAVEGUIDES}

84

We consider a three-layer waveguide placed in a free space, 85 as shown schematically in Fig. 1. Each layer $i$ has a thickness 86 $d_{i}$ and can have an arbitrary complex index of refraction. In the ${ }_{87}$ examples given below we assume that layers are of the same 88 thickness, $d_{i}=d$. We use the $\exp (-i \omega t)$ time convention, and 89 in this convention the positive imaginary part of the refractive 90 index describes lossy media, while negative values of this 91 quantity correspond to gain media. We look for TE-guided 92 modes, which have one nontrivial electric field component 93 $\left(E_{y}\right)$ and two magnetic field components $\left(H_{x}, H_{z}\right)$. Modes of 94 the structure have the form $E_{y}=E(x) \exp (i \beta z)$, where $\beta$ is 95 the mode wave number, and the mode profile $E$ is described 96 


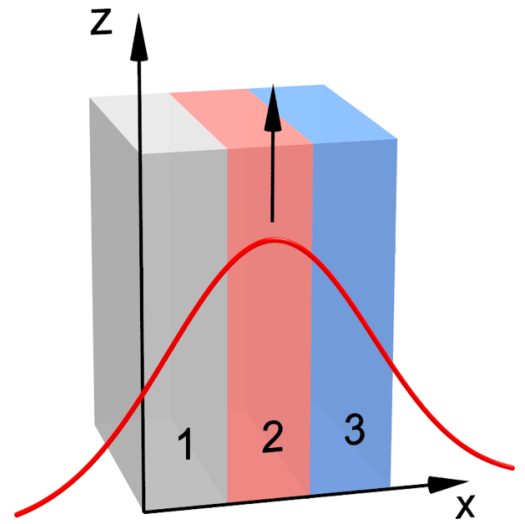

FIG. 1. Schematics of a three-layer non-Hermitian waveguide. Each layer can be either passive or exhibit gain or loss. For visual identification, we use red tint to denote gain layers, blue to denote loss layers, and grey to denote passive layers. by the equation

$$
\frac{d^{2} E}{d x^{2}}+\frac{\omega^{2}}{c^{2}}\left[\varepsilon(x)-\beta^{2}\right] E=0 .
$$

Following the standard procedure for the mode finding, 99 we write solutions in each layer and in the surrounding 100 vacuum, and in order to find the unknown constants we apply 101 the boundary conditions of the continuity of the tangential 102 components of the electric and magnetic fields. There are 103 eight unknown constants of integration and a set of eight linear 104 equations for these unknowns. The set of linear equations has 105 nontrivial solutions when the determinant of the matrix of the ${ }_{106}$ coefficients of this set vanishes. We explicitly write this matrix ${ }_{107}$ as

$\left.\begin{array}{cccc}0 & 0 & 0 & 0 \\ 0 & 0 & 0 & 0 \\ -1 & 0 & 0 & 0 \\ k_{2} & 0 & 0 & 0 \\ e^{-i k_{2} d_{2}} & -1 & -1 & 0 \\ -k_{2} e^{-i k_{2} d_{2}} & -k_{3} & k_{3} & 0 \\ 0 & e^{i k_{3} d_{3}} & e^{-i k_{3} d_{3}} & -1 \\ 0 & i k_{3} e^{i k_{3} d_{3}} & -i k_{3} e^{-i k_{3} d_{3}} & \kappa_{0}\end{array}\right]$

shown in Fig. 2(b) is shown in Fig. 3(a). It has a symmetric ${ }_{137}$ amplitude distribution, while the phase shows some gradient 138 indicating the energy flow from an active layer to a lossy layer. ${ }_{139}$

Cases B and C are quite remarkable, and they are offering $a \quad{ }_{140}$ new mechanism for controlling the required balance between 141 gain and loss in two nonconservative layers. Indeed, if we 142 have two layers of the same thickness, then the condition of ${ }_{143}$
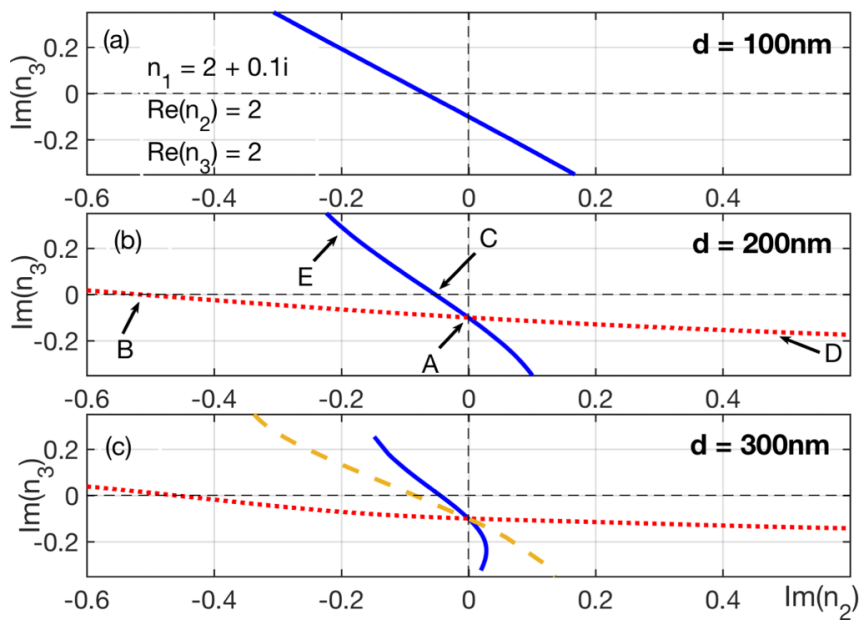

FIG. 2. Location of the energy-conserving modes on the plane of parameters of $\left[\operatorname{Im}\left(n_{2}\right), \operatorname{Im}\left(n_{3}\right)\right]$ for three different values of layer thickness $d$ : (a) $d=100 \mathrm{~nm}$, (b) $d=200 \mathrm{~nm}$, and (c) $d=300 \mathrm{~nm}$. In panel (b) points $\mathrm{A}, \mathrm{B}$, and $\mathrm{C}$ show the special cases, and points $\mathrm{E}$ and $\mathrm{D}$ correspond to general cases, which are discussed in the text. 

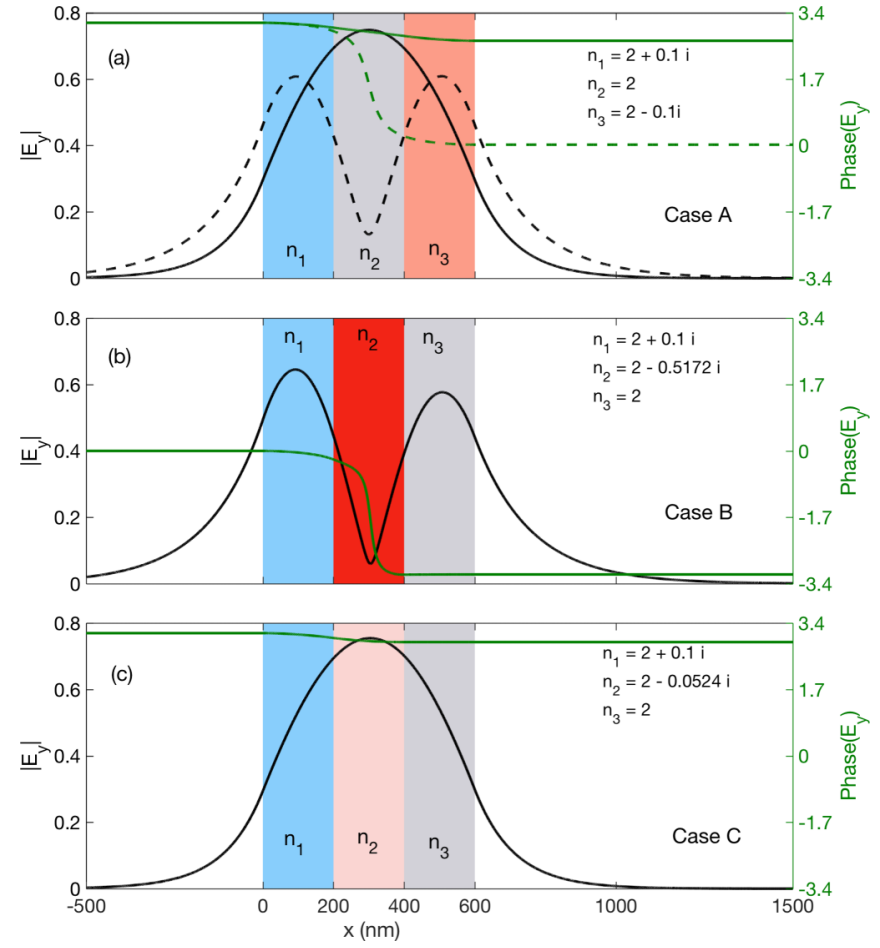

FIG. 3. Mode structure for three special cases; shown are the electric field amplitudes and phases for (a) a degenerate $\mathcal{P} \mathcal{T}$ symmetric case with parameters corresponding to point A in Fig. 1 and (b,c) two cases corresponding to points B and C in Fig. 1, when one of the layers is passive. Parameters of the structures are shown in the corresponding figure panels.

144 usual $\mathcal{P} \mathcal{T}$ symmetry requires that the amount of gain in one 145 of the layers is equal to the loss in another layer. Now, we ${ }_{146}$ can attach the third layer to the structure, and due to a change ${ }_{147}$ in the mode profile the amount of the required gain can be 148 either larger [case B, Fig. 3(b)] or smaller [case C, Fig. 3(c)]. 149 In the former case, the amount of gain is characterized be the 150 imaginary part of the index of refraction, $\operatorname{Im}\left(n_{3}\right) \approx-0.517$, 151 while in the latter case, it is -0.0524 , whose magnitude is 152 almost twice smaller than the loss coefficient $\operatorname{Im}\left(n_{1}\right)=0.1$. ${ }_{153}$ This is achieved by having larger field intensities in the gain 154 layer as compared to the field in the lossy layer.

155 Finally, in a more general case, the modes have a compli156 cated structure shown in Fig. 4, where we show two typical 157 modes corresponding to the two dispersion curves. One of 158 the modes resembles the fundamental mode of dielectric 159 waveguides with just one maximum, while another one is 160 double humped.

${ }_{161}$ Equation (3) has more than one solution. We study one 162 case of fixed parameters, case $\mathrm{E}: n_{1}=2+0.1 i, n_{2}=2-$ ${ }_{163} 0.2075 i$, and $n_{3}=2+0.3098 i$. In this case, we plot $\operatorname{det}(\hat{M})$ 164 on the complex plane of wave numbers in Fig. 5. We observe 165 that there are several zeros that correspond to the solutions of 166 Eq. (3). There is one solution that corresponds to the mode that 167 propagates without loss (marked by a red cross), and there are 168 multiple solutions with complex wave numbers corresponding 169 to the modes that decay away from the source. Thus, we can 170 conclude that our system provides energy conservation just for 171 one mode, whereas other modes experience attenuation.
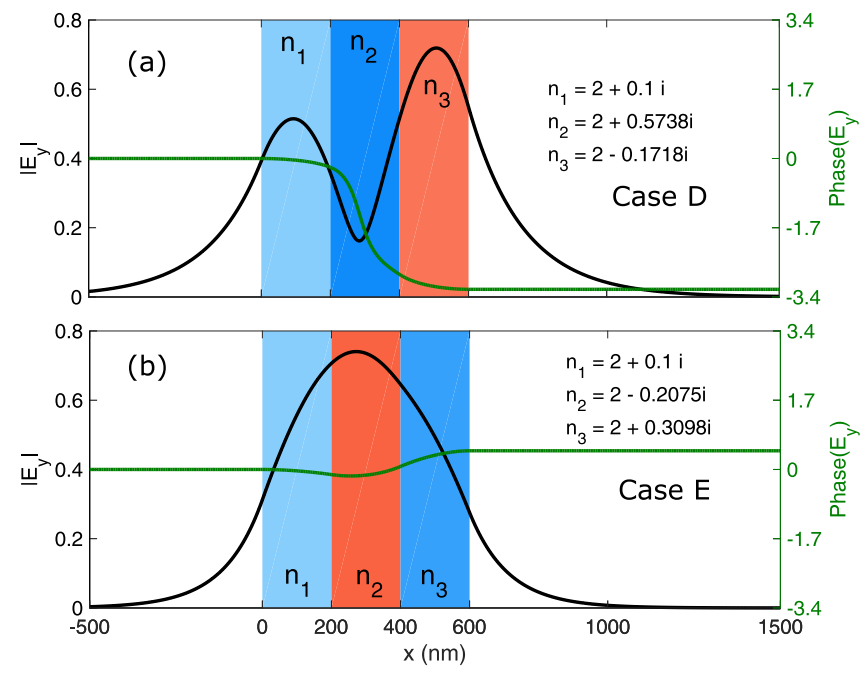

FIG. 4. Mode structure for two general cases. Shown are the electric field amplitudes and phases; (a) and (b) correspond to points $\mathrm{E}$ and $\mathrm{F}$ in Fig. 1, respectively.

Figure 6 shows the parameter plane of the imaginary parts $\left[\operatorname{Im}\left(n_{2}\right), \operatorname{Im}\left(n_{3}\right)\right]$ for the asymmetric case, when $\operatorname{Re}\left(n_{3}\right)=173$ 2.2, while $n_{1}$ and $n_{2}$ are the same as above. Two curves 174 corresponding to the two modes of the system still intersect at 175 one point, but this point is now not on the $\operatorname{Im}\left(n_{2}\right)=0$ axis, as it 176 was in the previously considered symmetric case. Remarkably, 177 this regime now possesses the same properties as the $\mathcal{P} \mathcal{T}-{ }_{178}$ symmetric case, i.e., both modes of the system have real eigen 179 wave numbers, but the system is not $\mathcal{P} \mathcal{T}$ symmetric. Thus, we 180 have revealed novel regimes in nonsymmetric structures when 181 all modes have their losses perfectly compensated by gain. $\quad 182$

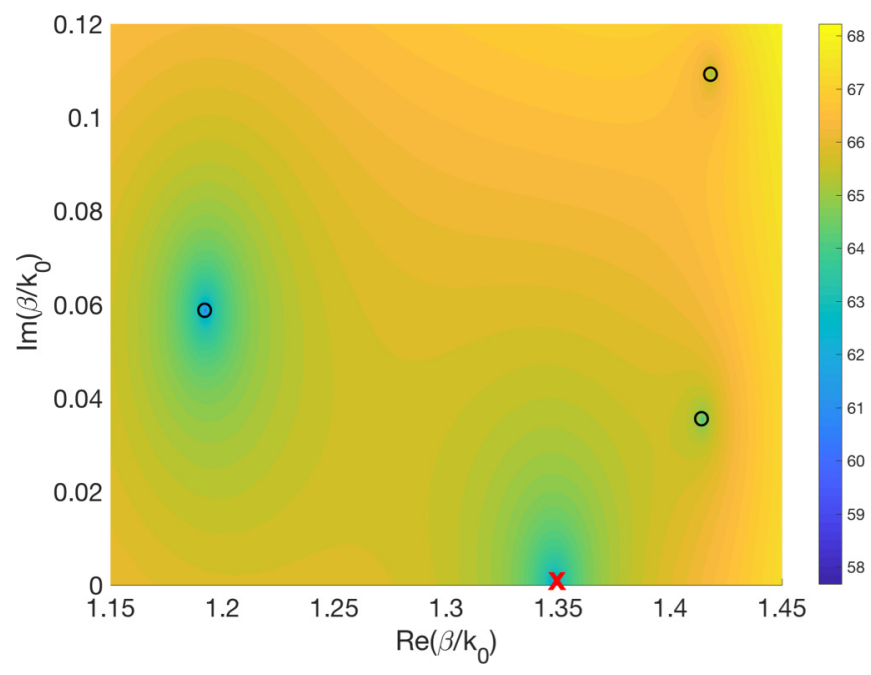

FIG. 5. Determinant of the matrix $M$ in the logarithmic scale on the plane of complex wave numbers. Shown are the points of the stationary propagating mode (marked by a red " $x$ ") and the nonpropagating modes (marked by black circles). 


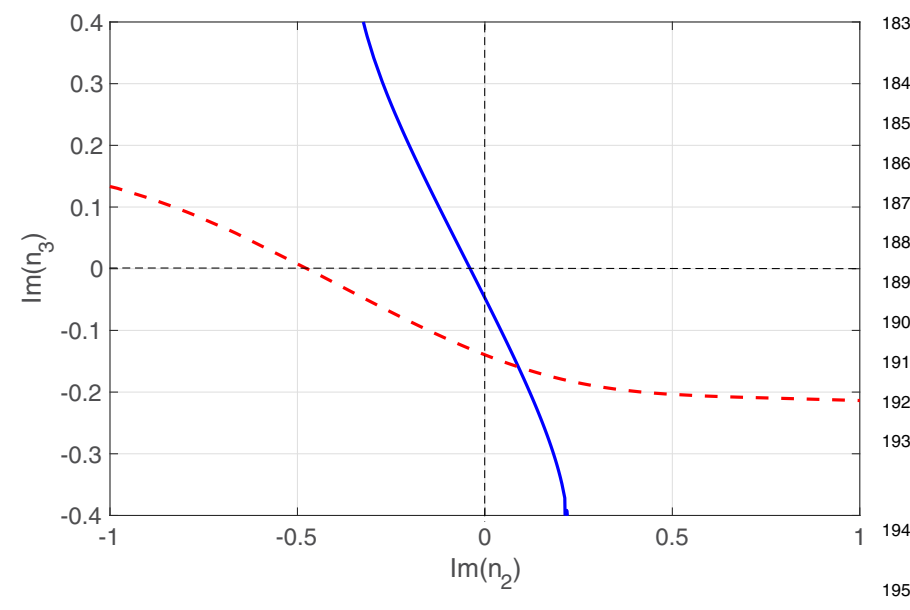

FIG. 6. Location of the energy conserving modes on the plane of parameters of $\left[\operatorname{Im}\left(n_{2}\right), \operatorname{Im}\left(n_{3}\right)\right]$ for the asymmetric case. Parameters are $d=200 \mathrm{~nm}, n_{1}=2+0.1 i, \operatorname{Re}\left(n_{2}\right)=2$, and $\operatorname{Re}\left(n_{3}\right)=2.2$.

\section{CONCLUSION}

We have studied the guiding properties of three-layer 85 non-Hermitian dielectric waveguides with gain and loss. We ${ }_{86}$ have revealed that the functionalities of conventional $\mathcal{P} \mathcal{T}$ 187 symmetric optical waveguides can be expanded substantially 188 by adding an additional dielectric layer and extending the 189 structure into a broader class of non-Hermitian systems to 190 control a ratio of gain and loss required to support propagating 191 and nondecaying guided modes. Our approach can be useful 192 for a design of novel types of waveguiding systems with 193 low-gain materials for the loss compensation.

\section{ACKNOWLEDGMENTS}

The authors acknowledge the support of the Australian Research Council and participation in the Erasmus Mundus NANOPHI project under Contract No. 2013 5659/002-001. 199 discussions and suggestions.
[1] T. Kato, Perturbation Theory for Linear Operators (Springer, Berlin, 1995).

[2] M. Schechter, Operator Method in Quantum Mechanics (Dover, New York, 2014).

[3] C. M. Bender and S. Boettcher, Phys. Rev. Lett. 80, 5243 (1998).

[4] C. M. Bender, Rep. Prog. Phys. 70, 947 (2007).

[5] F. Cannata, G. Junker, and J. Trost, Phys. Lett. A 246, 219 (1998).

[6] R. El-Ganainy, K. G. Makris, D. N. Christodoulides, and Z. H. Musslimani, Opt. Lett. 32, 2632 (2007).

[7] K. G. Makris, R. El-Ganainy, D. N. Christodoulides, and Z. H. Musslimani, Phys. Rev. Lett. 100, 103904 (2008).

[8] C. E. Ruter, K. G. Makris, R. El Ganainy, D. N. Christodoulides, M. Segev, and D. Kip, Nat. Phys. 6, 192 (2010).

[9] S. Savoia, G. Castaldi, and V. Galdi, Phys. Rev. A 94, 043838 (2016).

[10] V. Kovanis, J. Dionne, D. Christodoulides, and A. Desyatnikov, IEEE J. Sel. Top. Quantum Electron. 22, 0200502 (2016).
[11] S. V. Suchkov, A. A. Sukhorukov, J. Huang, S. V. Dmitriev, C. Lee, and Y. S. Kivshar, Laser Photon. Rev. 10, 177 (2016).

[12] C. Huang, F. Ye, and X. Chen, Phys. Rev. A 90, 043833 (2014).

[13] A. Mostafazadeh, J. Math. Phys. 43, 205 (2002).

[14] W. Walasik, C. Ma, and N. M. Litchinitser, New J. Phys. 19, 075002 (2017).

[15] A. E. Siegman, J. Opt. Soc. Am. A 20, 1617 (2003).

[16] B. Dastmalchi, P. Tassin, T. Koschny, and C. M. Soukoulis, Adv. Opt. Mater. 4, 177 (2016).

[17] A. Boltasseva and H. A. Atwater, Science 331, 290 (2011).

[18] S. Wuestner, A. Pusch, K. L. Tsakmakidis, J. M. Hamm, and O. Hess, Phys. Rev. Lett. 105, 127401 (2010).

[19] A. Fang, T. Koschny, and C. M. Soukoulis, Phys. Rev. B 82, 121102 (2010).

[20] H. Alaeian and J. A. Dionne, Phys. Rev. B 89, 075136 (2014).

[21] M. I. Stockman, Phys. Rev. Lett. 106, 156802 (2011).

[22] S. V. Suchkov, F. Fotsa-Ngaffo, A. Kenfack-Jiotsa, A. D. Tikeng, T. C. Kofane, Y. S. Kivshar, and A. A. Sukhorukov, New J. Phys. 18, 065005 (2016). 